How to reference this article

Mazzini, M. (2021). Bartolomeo Colleoni: un condottiero a cavallo di due lagune. Italica Wratislaviensia, 12(1), 197-214.

DOI: http://dx.doi.org/10.15804/IW.2021.12.1.11

\author{
Maurizio Mazzini \\ Wyższa Szkoła Bankowa we Wrocławiu, Polonia \\ maurizio.mazzini@wsb.wroclaw.pl \\ ORCID: 0000-0003-1432-6688
}

A Stettino, che è rimasta in me

\title{
BARTOLOMEO COLLEONI: UN CONDOTTIERO A CAVALLO DI DUE LAGUNE
}

\section{BARTOLOMEO COLLEONI: A MOUNTED LEADER SPANNING TWO LAGOONS}

\begin{abstract}
This article begins by broadly examining the history of the emergence and subsequent evolution of equestrian monuments, in both their commemorative and celebratory functions. Then, the article seeks to reconstruct the events leading to the construction of the mounted monument of Bartolomeo Colleoni, designed by Andrea del Verrocchio, and of the creation of its replica, still present in Szczecin, ordered in the early years of the $20^{\text {th }}$ century for the inauguration of the City Museum. The aim of the article is to present certain parallels in the destinies of the two artworks, both of which have fallen victim to politically motivated manipulations that result from efforts to "domesticate" the monument and erase traces of foreign influence upon the city. The origins of the very concept of equestrian monuments reach as far back as ancient times, while their sculptural typology encompasses art from geographically distant cultures, ranging from Europe to the Far East. In the Medieval period, the function of such monuments began to gradually shift, evolving from a commemorative and mnemonic one - related to funeral ceremonies and located in the burial places of the person immortalised in the sculpture itself - towards a more celebratory one, thus concluding a cycle that had begun in Antiquity. Throughout the Modern period, the monuments represented personas of the past characterised by particularly outstanding charisma and surrounded by an aura of virtue - personas that would embody the values propagated by the mercantile-focused bourgeoisie, as was the case with Szczecin's statue of Colleoni.
\end{abstract}

Keywords: Szczecin, Andrea del Verrocchio, Bartolomeo Colleoni, equestrian statues, mounted leader 


\section{IL MONUMENTO EQUESTRE DALLE ORIGINI AL QUATTROCENTO. CENNI STORICI}

Il seguente articolo si prefigge lo scopo di mostrare l'esistenza di determinate analogie, riscontrabili, malgrado la notevole distanza temporale e spaziale, fra gli avvenimenti legati alla nascita del monumento equestre a Bartolomeo Colleoni, opera di Andrea Verrocchio, ed il destino postbellico della sua copia stettinese, nonché di far luce sulle vicende che portarono alla realizzazione di quest'ultimo, episodio poco noto perfino in Polonia. Per far ciò si è ritenuto utile ripercorrere a grandi linee la storia del monumento equestre e delle sue funzioni e altresì inquadrare la nascita dell'originale e della sua copia nel contesto storico-culturale e politico in cui sono sorti.

Per monumento equestre si intende "la rappresentazione scultorea a tutto tondo di figure a cavallo, isolate o in gruppo" (Calcani, 1995, p. 5), una tipologia databile almeno a partire dal mondo greco-romano e che si dispiega nella vasta presenza del soggetto equestre in altri campi d'espressione artistica quali la scultura in miniatura e la pittura. Si tratta infatti di un soggetto fortemente ancorato nell'arte europea fin dall'Antichità, anche se non peculiare del nostro continente.

La fortuna dell'iconografia equestre va ricercata nella molteplicità funzionale di tale soggetto, che può essere finalizzato tanto alla raffigurazione di divinità quanto all'esaltazione di valori civici di una comunità o delle virtù individuali del personaggio raffigurato. Generalmente, si tratta di virtù connesse all'esaltazione della componente maschile della società: l'audacia, la destrezza con le armi, la fierezza. Lo dimostra il fatto che si tratta quasi sempre di personaggi maschili, e anche le rare eccezioni - come il monumento a Giovanna d'Arco di piazza des Pyramides a Parigi - si spiegano con la presunta mascolinità di carattere del personaggio.

Nella figura del cavaliere si concentrano non solo virtù atletiche, belliche e caratteriali, ma anche l'ostentazione della ricchezza, di cui il cavallo era status symbol, nonché il dominio quasi fisico della natura e degli istinti incarnati dall'animale, letteralmente e metaforicamente sottomesso. Una prassi che - come abbiamo già visto - collega gli euro- 
pei con i popoli delle steppe asiatiche, e si contrappone a quella in uso nella "mezzaluna fertile", dall'Egitto alla Mesopotamia, dove distintivo del rango sociale non è il destriero, bensì il carro su cui svetta, eretta, la figura di prestigio.

I primi esempi di statue equestri risalgono alla Grecia di epoca arcaica (VI secolo a. C.). In Attica si diffondono soprattutto i gruppi equestri in quanto forma monumentale eroicizzante, come nel cavaliere Rampin, marmo originariamente collocato sull'Acropoli, in cui si è voluto scorgere uno dei due figli di Pisistrato, ma la prassi doveva avere un raggio d'azione ben maggiore, se si considera, ad esempio, il cavallo rampante superstite rinvenuto a Locri e conservato nel reggino Museo Nazionale della Magna Grecia. Dal carattere rievocativo o festivo dei cortei di cavalieri che compaiono nei fregi del Partenone si passa a quello propagandistico della statuaria romana, volta a celebrare imperatori e capi militari viventi: la figura di Marco Aurelio ai Musei capitolini - scampata al "riciclaggio" perché interpretata come l'immagine del cristiano Costantino - e quella colossale di Traiano, pervenutaci, al pari di quella di Domiziano, solo in rappresentazioni numismatiche. Nella statuaria equestre imperiale domina il realismo del ritratto dal vivo, la precisione fisionomica che deve rendere riconoscibile al popolo il personaggio celebrato ${ }^{1}$, in contrapposizione alle figure idealizzate della scultura greca ed ellenistica.

L'arte bizantina in materia riprende gli stilemi di quella romana, come nella statua in bronzo di Giustiniano posta su colonna, andata distrutta con la conquista ottomana di Costantinopoli, per poi progressivamente svanire nella misura in cui la figura imperiale assume valenze sempre più religiose, divenendo così non rappresentabile.

A due importanti città, "capitali" italiane dell'Alto Medioevo, Pavia e Ravenna, sono riconducibili due statue equestri dedicate a Teodorico, entrambe andate perdute ${ }^{2}$, mentre al Louvre si conserva un piccolo

${ }^{1}$ Si tratta dunque di una funzione informativa, non dissimile da quella svolta dalle monete in circolazione nell'Impero, che univano funzione monetaria e propagandistica.

2 Originariamente la statua di Ravenna doveva rappresentare Teodorico, ma essa fu trasportata ad Aquisgrana per volere di Carlo Magno e qui adattata alle sembianze 
bronzo equestre datato intorno al IX-X secolo che raffigurerebbe Carlo Magno. L'avvento del Basso Medioevo segna a sua volta un regresso tecnologico abbinato all'impoverimento dei materiali utilizzati: la complicata tecnica della fusione del bronzo a cera persa cede il passo a materiali quali il legno, la pietra e, nella migliore delle ipotesi, il marmo. Ne sono fulgidi esempi i Cavalieri delle cattedrali di Bamberga e di Magdeburgo, realizzati in pietra e dedicati probabilmente a Federico II e Ottone I, in un clima di riviviscenza dell'arte classica che precede di due secoli il Rinascimento.

Un secolo dopo, la statuaria trecentesca, inaugura l'usanza di adornare con raffigurazioni equestri il monumento funebre dei signori dei nascenti stati regionali italiani. In quest'opera si distingue Bonino da Campione, a cui si deve sia il monumento a Bernabò Visconti in San Giovanni in Conca, oggi al Castello Sforzesco, sia l'ultima delle Arche scaligere, quella per Cansignorio, del 1375. Sempre in Veneto troviamo la prima statua equestre di un condottiero: nella basilica veneziana di Santa Maria Gloriosa dei Frari, riposa il romano Paolo Sanelli che offrì i suoi servigi alla Serenissima. L'opera, attribuita a Jacopo della Quercia, risale al primo ventennio del Quattrocento, secolo in cui, più di un capitano di ventura - Francesco Sforza in testa - assurge per meriti militari e ricchezza al prestigioso rango di duca. Capitani che vanno ad aggiungersi alla tipologia tradizionale, fatta di teste coronate, signori e rari Santi, come il San Martino a cavallo del Duomo di Lucca.

Se il monumento a Sanelli viene realizzato ancora in legno policromo, alla metà del Quattrocento si assiste alla rivincita del bronzo. Questo ritorno alla tradizione classica si sposa, in concomitanza con l'assurgere di Firenze a capitale del Rinascimento, con una precisa ripartizione geografia fra committenza ed esecuzione. I maggiori monumenti equestri rinascimentali saranno commissionati dalle Signorie del Nord Italia, ma affidati ad artisti toscani.

dell'imperatore in carica, riprendendo una prassi consueta nella scultura imperiale romana. 
Ad aprire la strada al ritorno all'utilizzo del bronzo a cera persa fu infatti il monumento eretto a Niccolò III d'Este, dei fiorentini Niccolò Baroncelli e Antonio di Cristoforo, con la supervisione di Leon Battista Alberti, appositamente chiamato a Ferrara da Lionello d'Este. In quest'opera, all'innovazione tecnologica si affianca una vera e propria rivoluzione funzionale: si tratta infatti del primo monumento equestre esclusivamente celebrativo e commemorativo, avulso dal contesto funebre e collocato non in un luogo sacro, bensì nelle immediate adiacenze di Palazzo Ducale, centro del potere politico, altra convergenza con la prassi diffusa nel mondo romano. Del resto il monumento si ispirava sia al cosiddetto "Regisole", fatto trasportare da Carlo Magno da Ravenna a Pavia che al Marco Aurelio. Purtroppo, come non di rado accadeva in un'epoca in cui l'artiglieria stava cambiando "l'arte della guerra", il bronzo venne fuso per esigenze belliche e successivamente sostituito in loco da una sua replica.

Un altro fenomeno tipico della seconda metà del XV secolo è la sinergia che si instaura fra pittura e scultura equestre in fase progettuale, annunciata dal dipinto di Paolo Uccello del 1436 in Santa Maria del Fiore che raffigura il condottiero Giovanni Acuto e maturata alla corte sforzesca, dove l'idea di Galeazzo Maria Sforza di far erigere una statua equestre in onore del padre, fondatore della signoria, viene ripresa da Ludovico il Moro. Nella lettera a lui rivolta con cui nel 1482 Leonardo cerca un impiego a Milano, l'artista, pur presentandosi soprattutto come ingegnere militare ed esperto di idraulica, si dichiara pronto a realizzare l'ambiziosa opera, rimasta a livello di pio desiderio, poiché non vi era nel ducato milanese persona capace di fondere in bronzo una statua equestre a grandezza naturale.

Prima di lui, un altro artista figurativo toscano, Antonio del Pollaiolo, aveva presentato la sua candidatura al progetto. $\mathrm{Ci}$ sono pervenuti due suoi progetti grafici (oggi a New York e a Monaco), posteriori di alcuni anni, in cui Francesco Sforza viene rappresentato sul cavallo impennato nell'atto di atterrare un fante. Quest'idea venne ripresa in un primo tempo da Leonardo, ma poi abbandonata, così come avverrà per l'altro progetto leonardesco non realizzato, quello del monumento funebre al maresciallo Gian Giacomo Trivulzio per San Nazaro a Milano. 
Leonardo riuscì a realizzare solo "l'anima" in creta dello Sforza, modello andato distrutto, e l'ardito progetto di Ludovico di creare una statua colossale restò lettera morta.

Quello che a Milano abortì, vide invece la luce a Padova, dove fra il 1446 e il 1453 Donatello aveva eretto il monumento a Erasmo da Narni, detto il Gattamelata. Anche Donatello prese a modello il Marco Aurelio, che si trovava all'epoca accanto alla basilica di San Giovanni in Laterano. Allo stesso modo, il Gattamelata, che per dimensioni è circa il doppio del perduto monumento a Niccolò III d'Este, fu collocato in terra consacrata, sul sagrato della Basilica del Santo e, a differenza del bronzo ferrarese, con funzione non solo celebrativa - fu finanziato dalla Repubblica di Venezia per cui il valoroso condottiero aveva combattuto - ma anche funebre, tanto che nei documenti viene indicato come "la sepoltura di Gattamelata", mentre due putti piangenti sul retro della sella ne sottolineano il contesto. Rispetto al modello romano, la statua si differenzia fra l'altro per l'armatura elaborata e la presenza delle staffe, micidiale invenzione, introdotta dai nomadi delle steppe asiatiche, che comunicano allo spettatore il senso del sicuro controllo dell'animale da parte del cavaliere. Ma ciò che più colpisce nella statua equestre di Erasmo da Narni è lo stupefacente naturalismo con cui è indagata la figura del cavallo, dove vene e muscoli non compromettono la plasticità dell'insieme. Come è stato acutamente osservato, essa costituisce la realizzazione figurata dell'immagine del cavallo delineata da Leon Battista Alberti nel suo De quo animante, di cui Donatello doveva essere a conoscenza. Del resto, proprio nel Rinascimento si andava delineando la "cultura" del cavallo, ormai largamente impiegato nei trasporti, nelle tecniche agricole, nei combattimenti, ma anche per diletto e per rappresentanza. Il suo elegante dominio diventa un requisito imprescindibile dei rappresentanti delle classi alte:

dalla metà del Quattrocento l'esercizio del cavalcare era sentito come parte fondante dell'educazione dei ceti abbienti e dei nobili e, parallelamente, acquisì valore e si consolidò il ruolo del maestro di equitazione, come era per il maestro di ballo e di musica. Nella pratica dei cavalieri aveva largo spazio il concetto di bellezza, di grazia, di armonia che animale e cavaliere attuavano insieme: movenze, andature, coreografia erano studiate e attuate in vista 
dell'ottenimento di uno spettacolo dove l'aspettativa della bellezza era alta.

(Baldissin Molli, 2011, pp. 146-148)

L'opera di Donatello si affermerà come modello di riferimento obbligato per la statuaria equestre successiva, dal Bartolomeo Colleoni del Verrocchio al Cosimo de' Medici del Giambologna, ma sarà proprio la statua equestre del bergamasco, con il suo dinamismo ormai cinquecentesco, a fare da ponte fra il Marco Aurelio e le statue equestri piacentine del Mochi.

\section{IL COLLEONI DEL VERROCCHIO}

Il comune denominatore che lega a prima vista le figure del Gattamelata e di Bartolomeo Colleoni, il fatto di essere stati entrambi soldati di ventura al servizio anche di Venezia, non deve sviarci dal considerare il contesto differente in cui nascono le due opere. In primo luogo, nel caso del monumento a Colleoni non si tratta di un mausoleo, bensì di un progetto di autocelebrazione del condottiero bergamasco stesso - sepolto nella città natale nella cappella omonima di Santa Maria Maggiore, il quale, in cambio di una cospicua somma di denaro lasciata in testamento allo Stato, da destinare alla guerra contro i Turchi, chiese esplicitamente che gli venisse eretto un monumento "davanti a San Marco". L'aspetto economico dell'operazione era dunque evidente, tanto più che, come scrive Ernst Gombrich, al Colleoni "i veneti erano legati da gratitudine più per alcune istituzioni benefiche da lui fondate che per atti di valore" (Gombrich, 1995, p. 293). Tuttavia, la volontà del defunto fu rispettata solo in parte: nel 1495, a quindici anni dall'affidamento dell'incarico al Verrocchio, la statua sarà collocata in Campo Santi Giovanni e Paolo. Si tratta, a dire il vero, di un'ubicazione di prestigio, dal momento che l'omonima chiesa domenicana costituisce il pantheon delle glorie di Venezia, ma non così centrale come voleva il benefattore. Pare che il governo veneziano, di fronte al divieto di collocare monumenti in piazza San Marco, abbia giocato sull'imprecisione della disposizione testamentaria, interpretandola liberamente come "davanti alla Scuola Grande di San Marco". In secondo luogo, mentre il Gattamelata è interamente opera di 
Donatello, il Colleoni fu fuso in bronzo dal fonditore locale Alessandro Leopardi, dopo che l'artista toscano era deceduto nel 1488. Nondimeno, stando all'opinione di Gombrich, il valore e l'influsso del monumento veneziano non sono inferiori a quelli del suo predecessore padovano:

La statua equestre mostra come il Verrocchio sia un degno erede della tradizione di Donatello. Vediamo con quale minuzia abbia studiato l'anatomia del cavallo, il gioco dei muscoli del collo e della faccia di Colleoni; ma la cosa più mirabile è l'atteggiamento del cavaliere, che sembra caracollare alla testa delle truppe, con un'espressione di ardimentosa sfida. I tempi più recenti ci hanno reso tanto familiari questi cavalieri di bronzo, venuti a popolare le nostre città con raffigurazioni di imperatori, re, principi e generali più o meno degni, che stentiamo a renderci conto della grandiosità e della semplicità dell'opera del Verrocchio, del profilo netto che il suo gruppo presenta quasi da ogni lato e dell'energia concentrata che pare animare l'uomo nell'armatura e il destriero. (ibid.)

Rispetto all'opera di Donatello, mutate sono le proporzioni fra cavaliere e cavalcatura a vantaggio del primo, che domina la composizione. La pacata classicità della posa del Gattamelata, il suo sereno incedere, lasciano il posto all'esaltazione del carattere drammatico, basato sulla plasticità chiaroscurata del Colleoni. La sua maggiore dinamicità scaturisce dall'incrocio della diagonale formata dal dorso e dalla criniera del cavallo con quella disegnata dalla figura del condottiero e della zampa sinistra dell'animale. La tensione muscolare dell'animale, unita allo scatto in avanti della spalla sinistra e all' espressione corrucciata del volto conferiscono al monumento un carattere più bellicoso, lo impregnano di energia vitale. Anche l'assenza della sfera sotto lo zoccolo anteriore del cavallo, che nel monumento padovano svolgeva funzione di equilibrio al tempo stesso statico e compositivo, esalta la sensazione che il gruppo monumentale sia proiettato in avanti, con un vigore che incute timore: nella società civile e nella politica italiana nuvole minacciose si stanno addensando all'orizzonte e l'arte registra quella crescente inquietudine che culminerà alla fine del secolo con gli sconvolgenti affreschi sul Giudizio Universale di Luca Signorelli nella cappella Brizzi del Duomo di Orvieto: se l'opera di Donatello si colloca cronologicamente nel periodo contrassegnato dalla politica d'equilibrio voluta da Lorenzo il Magnifi- 
co, quella del Verrocchio e del suo successore si situa in un momento in cui si sta scivolando ineluttabilmente verso la fine del primato politico e culturale degli stati regionali, che sfocerà nelle Guerre d'Italia.

La vicenda del monumento ebbe inizio il 30 luglio 1479, a quattro anni dalla morte del condottiero, quando la Serenissima ne decretò la costruzione. L'anno seguente, l'esecuzione fu affidata ad Andrea del Verrocchio, che intraprese la realizzazione del modello in cera nella sua bottega fiorentina. Trascorso un altro anno esso venne inviato a Venezia, dove l'artista si trasferì nel 1486 per attendere alla fusione in cera persa del bronzo. Due anni dopo però il Verrocchio morì proprio - stando al Vasari - a causa di quel lavoro, peraltro incompiuto: "essendo riscaldato e raffreddato nel gettarlo" (Vasari, 2015, p. 381), lasciando in eredità al suo successore un modello in creta. Questi, secondo il testamento del fiorentino, doveva essere il suo concittadino e allievo di bottega nonché esecutore testamentario Lorenzo di Credi, più noto come pittore che non come scultore. Così come era avvenuto nel caso di Colleoni, la Repubblica di Venezia non rispettò la volontà testamentaria di un forestiero, affidando la prosecuzione dell'opera all'artista locale Alessandro Leopardi. Del resto i rapporti fra la Serenissima e il Verrocchio erano stati turbolenti fin dai primi tempi, al punto che l'artista aveva piantato baracca e burattini e fatto ritorno a Firenze, suscitando l'ira dei suoi committenti, placata solo dall'arguzia con cui lo scultore aveva reagito alle minacce della Serenissima (cf. ibid.).

Il nuovo esecutore, maestro di zecca e architetto, realizzò la fusione in bronzo malgrado le difficoltà dovute alle dimensioni del gruppo equestre che sfiora i quattro metri di altezza. A ricordo di quell'impresa leggiamo l'orgogliosa firma dell'artista, posta sotto il ventre del cavallo: ALEXANDER LEOPARDUS V. F. OPUS. Il 21 marzo del 1496 l'opera venne inaugurata, suscitando grande curiosità di pubblico. Qualche mese prima essa era stata collocata su un alto basamento con colonne da Leopardi stesso progettato, avente lo scopo di simboleggiare il massimo riconoscimento da parte della Repubblica per il personaggio rappresentato. Non è difficile scorgere nell'affidamento dell'esecuzione dell'opera ad un artista veneziano non tanto la volontà di valorizzare l'ambiente artistico locale, quanto un tentativo di appropriazione politica dell'o- 
pera, di purgarlo il più possibile degli influssi forestieri, in particolare fiorentini, visti i non facili rapporti che da decenni intercorrevano fra Venezia e Firenze ${ }^{3}$.

\section{IL COLLEONI STETTINESE}

Anche Stettino, come Venezia, ha la sua laguna, un braccio di mare che si incunea nella costa della Pomerania per una settantina di chilometri, e al cui termine, in prossimità della foce dell'Oder è sorta la città. Il suo passato commerciale risale al Medioevo, quando essa, in virtù della sua posizione privilegiata si sviluppa come città anseatica. Tuttavia, è solo verso la fine del Settecento, in seguito alla sua annessione alla Prussia che si gettano le basi del suo successivo sviluppo in senso capitalistico. Il costituirsi di un vasto retroterra economico fa sì che Stettino diventi nel corso di un secolo il principale porto del Regno di Prussia. Il periodo che intercorre fra la metà dell'Ottocento e la Prima Guerra Mondiale rappresenta quindi il secolo d'oro della città. Lo sviluppo delle comunicazioni sia terrestri che navali fa di Stettino un importante centro commerciale e industriale: al ruolo di porto di transito delle merci, si affianca quello produttivo, specie nel campo della cantieristica, della produzione del cemento e dell'industria agroalimentare, che si avvaleva anche dei prodotti provenienti dalla regione polacca della Grande Polonia.

L'aumento della ricchezza complessiva, a partire dalla fine del Settecento, si traduce in un notevole incremento demografico che porta al raddoppio del numero degli abitanti nel corso della prima metà del secolo successivo. La Città Vecchia, arroccata attorno al Castello dei Principi di Pomerania, e costretta dalla cinta muraria, diviene troppo angusta e alla metà dell'Ottocento vi si affianca la Città Nuova, ma è proprio a cavallo fra Ottocento e Novecento che prende forma l'assetto urbanistico che ancora oggi caratterizza Stettino: la presenza di grandi piazze,

3 Venezia aveva concesso ospitalità agli esuli fiorentini, primo fra tutti Cosimo il Vecchio, riparato a Venezia con tutti i suoi averi nel 1433. Nel 1466 la Serenissima concesse asilo anche a Niccolò Soderini e Diotisalvi Neroni, congiurati al servizio di Luca Pitti contro Piero de' Medici, detto il Gottoso. 
da cui si diramano a raggera le vie principali, un modello che sembra imitare le ambizioni urbanistiche di una metropoli europea in ascesa, la Parigi di Eugène Haussmann.

A coronamento di quest'opera di ridefinizione e arricchimento simbolico dello spazio urbano si colloca la costruzione, nei primissimi anni del Novecento (1901-1905), della Hakenterrasse (oggi Argini di Boleslao l'Ardito), un'infilata di edifici monumentali, tra storicismo ed eclettismo, voluta del sindaco Hermann Haken, che dominano la foce dell'Oder e disegnano una promenade con vista sul porto, quasi a voler bearsi visivamente della propria opulenza ed operosità, e che ancora oggi costituisce il biglietto da visita della città. I tre edifici che la compongono riassumono simbolicamente la vocazione della Stettino dell'epoca: commerci, ordine e cultura si materializzano rispettivamente nei due palazzi congiunti dei Dazi e delle Assicurazioni, nella Prefettura della Regione Stettinese e nel Museo Civico, eretto negli anni 1907-1913, dove trovarono fra l'altro posto le collezioni museali offerte dalla famiglia Dohrn (cf. Białecki, 2015, p. 156). L'appartenenza alla Prussia non tragga in inganno: la borghesia stettinese a cavallo del secolo ha poco della grettezza mentale e della limitatezza d'orizzonti che in genere si attribuisce allo stato militarista prussiano: essa guarda più davanti a sé, alla Svezia, che non alla Berlino situata alle sue spalle. Una famiglia come quella dei Dohrn, unisce vasti interessi culturali e scientifici con l'attività mercantile di importatori di prodotti esotici e con quella industriale. Se il capostipite, Heinrich, importa spezie, vino e agrumi, produce zucchero, creando una delle prime S.p.A. prussiane, il figlio, Carl August, raccoglie non solo farfalle in vari continenti e dà vita alla prima Associazione Entomologica tedesca, ma colleziona opere d'arte, tanto europee, quanto africane, mentre suo figlio, Heinrich Wolfang Ludwig Dohrn (1838-1913), uomo politico e industriale, arricchisce le collezioni del padre e fonda la prima Associazione Museale della Pomerania, senza per questo trascurare le sue piantagioni di tabacco a Sumatra, il che la dice lunga sulla versatilità e sul raggio d'azione della borghesia stettinese.

Sebbene gli inizi dell'attività museale a Stettino risalgano al 1821 e al gabinetto di scienze naturali esistente presso il Ginnasio della Fon- 
dazione Mariana, fu proprio la famiglia Dohrn a segnare una svolta nella storia dei musei stettinesi. Nel 1863 Heinrich Wolfang Ludwig Dohrn, figlio del già menzionato eminente entomologo Carl August, propose di fondare il Museo della Pomerania, dedicato alle scienze naturali e trent'anni dopo, nel 1892, volendo raccogliere in un solo edificio le varie collezioni museali della città fece dono a questa delle collezioni del padre, appena defunto, e della casa di famiglia di Lindenstrasse (oggi ul. 3 Maja) affinché fosse destinata a sede del Museo Civico di cui divenne direttore, alle quali andarono ad aggiungersi, a pochi anni di distanza, le collezioni del Museo della Pomerania, ormai chiuso. Il gruppo di entusiasti che nel 1910 porterà ad istituire 1'Associazione Museale Stettinese (Stettiner Museumsverein) riuscì ad ottenere l'appoggio delle autorità municipali, che nel 1900 deliberarono di erigere un nuovo monumentale edificio dove poter riunire definitivamente le raccolte museali sparse per la città. Tale edificio fu innalzato negli anni 1908-1913, nella parte centrale della "Terrazza di Haken", su progetto di Wilhelm Meyer, architetto comunale, ideatore dell'intero complesso, che tuttavia fu realizzato solo a metà, malgrado la raccolta di fondi durata anni ed anni. Il nuovo Museo Civico (di cui l'odierno Museo Nazionale è almeno in parte l'erede), monumentale e pre-modernista, possedeva una serie di ampie sale nonché una grande hall a due piani, posta sotto la cupola centrale (oggi appartenente al Teatro Contemporaneo). L'edificio venne inaugurato nel 1913, alla vigilia di quella Prima Guerra Mondiale che avrebbe segnato l'inizio di una crisi da cui la città prussiana si sarebbe risollevata parzialmente solo grazie agli investimenti a fini bellici operati dall'amministrazione nazista, per poi sprofondare sotto i colpi dell'artiglieria sovietica.

Proprio in questo spazio privilegiato trovò degna collocazione la collezione di copie d'arte antica e rinascimentale di Heinrich Wolfang Ludwig Dohrn, avviata con una mostra organizzata nel 1904 in un ginnasio cittadino e comprendente fra l'altro copie di reperti archeologici provenienti da Pompei ed Ercolano. In seguito, con il sostegno di vari sponsor egli diede vita ad una galleria contenente 120 copie di statue greche ricostruite nel materiale originario. Ora, il nuovo Museum der Stadt Stettin, tipica espressione del collezionismo borghese della belle époque, gli offriva l'opportunità di collocare la sua raccolta in un edifi- 
cio capace di contenere e presentare adeguatamente le radici della cultura europea, realizzando così uno dei suoi sogni. La collezione Dohrn fu arricchita con la copia della statua equestre del Colleoni, eseguita con la tecnica della galvanoplastica che consiste nel ricoprire, attraverso l'elettrolisi, con un metallo un materiale non conduttore. In pratica si trattava di produrre in zinco i frammenti di cui è composta, saldarli, rinforzare la struttura internamente vuota con un'impalcatura d'acciaio e infine rivestire la statua di bronzo con la tecnica galvanica. L'incarico venne affidato alla "Fabbrica di Prodotti in Metallo del Württemberg", in località Geislingen, nei pressi di Stoccarda, nel 19094. A finanziare l'iniziativa $\mathrm{fu}$, attraverso la fondazione di famiglia, un altro celebre rappresentante dell'imprenditoria stettinese dell'epoca, il conoscente di Dohrn nonché consigliere comunale Albert Töpffer, detto "il re del cemento", dopo che - stando a quanto riportò la stampa stettinese nel giugno del 1911 il suo primogenito, il dr Helmut Töpffer e la consorte Helse Grawitz, avevano espresso l'intenzione di commemorare la famiglia di quest'ultima con alcune copie di opere rinascimentali (cf. Leśniak \& Mróz, 1997, p. 23)

La statua del condottiero bergamasco fece dunque bella mostra di sé, quel 23 giugno 1913, il giorno dell'inaugurazione del nuovo Museo Civico. Essa avrebbe dovuto trovarsi in compagnia della replica di un'opera d'arte del Rinascimento italiano, il Mosé di Michelangelo, ideato per il monumento funebre a papa Giulio II, oggi a San Pietro in Vincoli, ma questa volta l'idea non andò in porto: il nuovo direttore Walter Riezler non la condivideva e la statua in marmo fu collocata nella stanza rinascimentale della villa appartenente al committente stesso, oggi scomparsa, nel quartiere di Stettino-Zdroje, in prossimità del suo cementificio.

${ }^{4}$ Come scrivono Janusz Krause e Janusz A. Mróz, "la produzione delle copie galvanoplastiche veniva perfettamente incontro ai bisogni del mercato. Gli abitanti delle città, che diventavano sempre più ricchi, e desideravano circondarsi di opere d'arte, potevano in tal modo realizzare le loro aspirazioni, poiché, ovviamente, solo pochi di loro avrebbero potuto acquistare opere originali" (Krause \& Mróz, 2008, p. 84) (trad. propria). 
Che la scelta sia caduta sul Colleoni e non sul Gattamelata non stupisce: la figura del condottiero bergamasco meglio rispondeva alla weltanschauung della rapace e rampante borghesia imperialistica stettinese. Per dirla con le parole dello storico dell'arte Raffaele De Grada: "Se il Gattamelata era stato il condottiero che aveva fatto la guerra per conquistare la pace, il Colleoni è il guerriero che fa paura" (De Grada, 1977, p. 41). Del resto, non desta sorpresa l'accostamento di Stettino con Venezia, città che dovevano entrambe la loro fortuna alla navigazione e al commercio. Anche l'aver commissionato una copia del Mosé di Michelangelo può essere letto in questa chiave: in fondo fu proprio lui, fra i personaggi biblici, quello che, con l'aiuto divino, fu capace di domare le acque del mare e piegarle al suo volere.

Se nel caso del Colleoni veneto possiamo parlare di peripezie in fase di realizzazione, per quanto riguarda quello pomerano esse ebbero inizio con la rinascita di Stettino nelle vesti di città polacca. L'incertezza sull'assegnazione definitiva delle cosiddette terre riconquistate, dei territori di Pomerania, Slesia e Prussia Orientale strappati dall'Armata Rossa alla Germania nazista, fece sì che per decenni gli investimenti in queste regioni fossero ridotti al minimo, mentre procedeva l'opera di smantellamento degli impianti e delle infrastrutture trasportabili superstiti, avviata immediatamente dopo la fine delle ostilità. Una politica di drenaggio delle risorse, per non dire di rapina, conclusasi non prima degli anni Settanta del Novecento, con il riconoscimento da parte della Repubblica Federale Tedesca della frontiera sull'Oder-Neisse.

Una sorte simile è toccata a numerose opere d'arte "messe al sicuro" nella capitale. E così, il 15 gennaio 1948 anche il nostro Colleoni, dopo essere stato ritrovato in pezzi dall'Armata Rossa, imballato in casse, in una delle foreste nei pressi della città dove era stato evacuato, fu affidato al prof. Stanisław Lorentz, direttore del Museo Nazionale di Varsavia, e trasportato nella capitale, dove trovò inizialmente posto nel cortile del Museo stesso, a Palazzo Raczyński, luogo evidentemente troppo prestigioso, tanto che venne presto spostato negli scantinati del Museo dell'Esercito Polacco e solo nella primavera del 1950 rivide la luce nel cortile dell'Accademia di Belle Arti, la stessa 
scuola a cui approdò la statua del Mosè, entrambi in qualità di "doni" offerti da Stettino alla capitale che risorgeva dalle macerie ${ }^{5}$. Si noti che in certi casi, come in quello del Colleoni, venivano prelevate opere che non avevano ancora lo status di "monumento" (Colleoni sarà registrato come tale solo nel 1989), il che fa sorgere il sospetto che si trattasse più di sradicarle dal loro habitat culturale originario che non di metterle al sicuro in vista di una possibile revisione delle frontiere. Che gli stessi varsaviani si rendessero conto dell'estraneità culturale del monumento alla capitale ce lo conferma lo storico e archivista Eugeniusz Szwankowski, il quale scrisse su Stolica nel 1949: "Per Varsavia è ovviamente una figura indifferente e pertanto non può abbellire nessuna piazza a carattere rappresentativo né alcuna via e deve essere trattata solo come un'opera d'arte" (Leśniak \& Mróz, 1997, p. 23).

Finalmente, nel 1992, nel nuovo clima politico, in seguito ad un'iniziativa popolare sfociata nella richiesta ufficiale di restituzione del monumento inoltrata dal Museo Nazionale di Stettino, con l'appoggio del sindaco Edmund Runowicz, fu avviata la complicata procedura che avrebbe consentito alla comunità stettinese, in cui stava germogliando l'idea della indivisibilità della storia della città in "nostra" e "loro", il recupero del monumento equestre. Ironia della sorte, il condottiero che più di uno fece prigioniero dovette essere riscattato con il finanziamento di una copia in bronzo della copia, da lasciare a Varsavia, per permettere alla "copia originale" di far ritorno in Pomerania. Metà dei 350.000 złoty necessari per liberarlo fu versata dal Comune, mentre la restante parte venne raccolta grazie ad una sottoscrizione popolare. Solo nel 2002 il monumento fece ritorno a Stettino, dove è stato collocato in piazza Lotników, risistemata per l'occasione. A dimostrazione della cura con cui fu organizzata la cerimonia inaugurativa, sta il fatto che vi prese la parola un discendente del condottiero, venuto appositamente da Bergamo (cf. Szymków, 2002, p. 8). Quanto al suo compagno di sventura, il Mosé "michelangiolesco", si sarebbe riusciti a riscattarlo dall'Accademia varsaviana soltanto nel 2017, grazie ai fondi europei

${ }^{5}$ L'iniziativa partì dal cosiddetto Comitato di Aiuto alla Capitale, ma è probabile che fosse pilotata dall'alto. 
destinati alla costituzione del "Museo delle Tradizioni Regionali", con cui Stettino poté rientrare in possesso del dono che aveva fatto.

Purtroppo, la riappropriazione in toto della memoria storica della città, nel bene e nel male, tanto a Stettino quanto in altre città polacche, si fa strada a fatica, fra riserve di natura mentale e strumentalizzazione politica. Negli ultimi anni si assiste infatti in Polonia al rigurgito di quella ideologia "panpolacca" e antitedesca che aveva caratterizzato la Polonia Popolare e che ora viene rilanciata dalla destra nazional-cattolica al potere. Come scrive Bartosz Wójcik,

nel periodo della Repubblica Popolare Polacca si cercò di costruire una nuova identità della città e della regione; uno dei principali elementi di tale identità era costituito da una nuova narrazione storica che sottolineava i legami fra la Pomerania Occidentale e la Polonia dei Piasti e al contempo sosteneva la tesi del declino economico della regione nel periodo prussiano e tedesco. A questa narrazione si legava la negazione dell'eredità culturale risalente all'epoca tedesca. (Wójcik, 2017, p. 163)

È dell'autunno del 2019 l'idea di un consigliere comunale stettinese di far sloggiare il condottiero dalla sua attuale ubicazione, per far posto alla statua del fondatore della patria Józef Piłsudski, ora in piazza Szarych Szeregów, poiché, a suo avviso, a Stettino mancherebbero piazze altrettanto adatte per organizzare manifestazioni patriottiche l'11 novembre, festa nazionale. Gli fa eco la dichiarazione surreale dell'Associazione degli Appassionati di Piłsudski di Gorzów Wielkopolski in reazione all'idea di disarcionare il condottiero, collocando il patriota polacco sul cavallo del bergamasco: "Associare il grande uomo di Stato Józef Piłsudski con l'assassino italiano, Bartolomeo Colleoni è deplorevole; il cavallo in alcun modo non assomiglia alla nobile cavalla da battaglia del Maresciallo Józef Piłsudski, detta "Castagna".

Verrebbe da dire che il clima lagunare non giovi al nostro condottiero. La Serenissima fece di tutto, a più riprese, per appropriarsi dell'opera

${ }^{6}$ Con queste parole l'associazione si è rivolta al Comune di Stettino in merito al possibile trasferimento della statua dell'uomo di stato polacco. Citazione da Gazeta Wyborcza (edizione stettinese) del 18.10.2019. 
d'arte e per così dire "venezianizzarla", farla attecchire nel proprio habitat culturale e politico, e ci riuscì, anche grazie alla prematura scomparsa dello scultore toscano; le autorità della Repubblica Popolare Polacca, applicando una logica almeno altrettanto perversa, e in fin dei conti convergente negli scopi, hanno cercato, al contrario, di sradicarla dal suo contesto, identificandola come parte del patrimonio dei vinti, radici tedesche che non si voleva affiorassero dalla nuova patina da applicare alla città "riconquistata" e pertanto andavano estirpate.

È consolante il fatto che, malgrado i pericoli a cui entrambe sono attualmente esposte, l'acqua alta dell'Adriatico e l'onda lunga del nazionalismo, le due incarnazioni del condottiero bergamasco siano ancora al loro posto, mantenendo in vita questo curioso parallelismo venetopolacco.

\section{BIBLIOGRAFIA}

Baldissin Molli, D. (2011). Erasmo da Narni - Gattamelata e Donatello. Padova: Centro studi antoniani.

Białecki, T. (2015). Encyklopedia Szczecina. Szczecin: Szczecińskie Towarzystwo Naukowe.

Calcani, G. (1995). Monumento equestre. In Enciclopedia dell'arte antica. Roma: Treccani.

Calcani, G. (1989). Cavalieri di bronzo: la torma di Alessandro opera di Lisippo. Roma "L'Erma" di Bretschneider.

Cornazzano, A. (1990). Vita di Bartolomeo Colleoni. Manziana-Roma: Vecchiarelli.

De Grada, R. (1977). Storia dell'arte, vol. 3: L'epoca moderna. Napoli: Morano.

Gombrich, E.H. (1995). La storia dell'arte raccontata da E.H. Gombrich. Roma: Leonardo Libri.

Jakóbik, M. (2011). Plac Tobrucki - reprezentacyjny zieleniec przedwojennego Szczecina. Renowacje i zabytki, 4(40), 102.

Krause, J., \& Mróz, J.A. (2008). Kopia konnego pomnika Bartolomea Colleoniego. Zagadnienie eksponowania kopii dzieł sztuki. Wiadomości Konserwatorskie, 23, 81-93.

Leśniak, K., \& Mróz, J.A. (1997). Kopia weneckiego pomnika kondotiera Bartolomea Colleoniego. Cenne, Bezcenne, Utracone, 5, 23. 
Szymków, M. (2002, August 30). To było kompletne zaskoczenie. Potomek Bartolomea Colleoniego w Szczecinie. Gazeta Wyborcza Szczecin, 202, 8.

Vasari G. (2015). Le vite dei più eccellenti pittori, scultori e architetti. Roma: Newton Compton.

Wójcik, B. (2017). Sedina i Colleoni - szczecińskie implanty pamięci społecznej. In D. Gortych, \& Ł. Skoczylas (Eds.), Implanty pamięci społecznej. Teoria i przykłady (pp. 161-180). Poznań: Rys.

Riassunto: Dopo aver ripercorso a grandi linee le vicende della nascita e dello sviluppo del monumento equestre, nelle sue funzioni commemorativa e celebrativa, l'articolo ricostruisce le fasi che portarono alla realizzazione della statua a cavallo veneziana dedicata a Bartolomeo Colleoni, su progetto di Andrea del Verrocchio, e della sua copia tuttora esistente a Stettino, ordinata nei primi del Novecento per l'inaugurazione del Museo Civico. Lo scopo è quello di dimostrare l'esistenza di un certo parallelo nel destino delle due opere, entrambe vittima di manipolazioni di tipo politico, incentrate sulla volontà di "addomesticare" l'opera d'arte e cancellare le tracce dell'influsso straniero sulla città. Le origini del monumento equestre sono antichissime e tale tipologia scultorea contraddistingue culture anche geograficamente lontane, dall'Europa all'estremo oriente. Nel Medioevo la sua funzione cambia progressivamente, evolvendosi da quella commemorativa, legata alla cerimonia funebre e al luogo di eterno riposo della figura rappresentata verso quella celebrativa, chiudendo così quel ciclo che si era aperto nell'Antichità. In epoca moderna, personaggi del passato, dotati di particolare carisma e avvolti da un'aura di virtù, potevano incarnare i valori propugnati dalla borghesia mercantile, come nel caso del Colleoni stettinese.

Parole chiave: Andrea del Verrocchio, Bartolomeo Colleoni, statua equestre, Stettino, condottiero 